\title{
Inhibition of autophagy enhances the radiosensitivity of nasopharyngeal carcinoma by reducing Rad51 expression
}

\author{
NING MO*, YONG-KUI LU*, WEI-MIN XIE*, YAN LIU, WEN-XIAN ZHOU, HONG-XUE WANG, LI NONG, \\ YU-XIAN JIA, AI-HUA TAN, YING CHEN, SHAN-SHAN LI and BAO-HUA LUO
}

The Fifth Department of Chemotherapy, Affiliated Tumor Hospital of Guangxi Medical University, Nanning 530021, P.R. China

Received April 28, 2014; Accepted July 20, 2014

DOI: $10.3892 /$ or.2014.3427

\begin{abstract}
Radiotherapy has long been considered as the mainstay of treatment for nasopharyngeal carcinoma (NPC). However, locoregional recurrence or distant metastasis may occur in some patients due to the radiation resistance of cancer cells. Autophagy plays a vital role in protecting cells against radiation. However, the mechanism of autophagy in radiation therapy remains obscure. In the present study, we demonstrated that suppression of autophagy related 5 (Atg5) aggravated ionizing radiation (IR)-induced DNA damage and apoptosis in human NPC cells without accelerating the cell cycle, whereas regulation of the cell cycle has been widely regarded as the most important determinant of IR sensitivity. Further study showed that inhibition of autophagy suppressed the mRNA expression of Rad51, a key protein of homologous recombination that has been demonstrated to play a critical role in the repair of DNA double-strand breaks induced by radiation. Moreover, suppression of Atg5 had no impact on the radiosensitivity when cells were pre-treated by the Rad51 inhibitor, and the enhanced radiosensitivity by Atg5 suppression was reversed by overexpression of Rad51 in human NPC cells. Our results suggest that inhibition of autophagy enhances the susceptibility of NPC cells to radiation by reducing Rad51 expression. Therefore, Rad51 targeted therapy may be investigated as a potential novel agent for the adjuvant treatment of traditional radiation of NPC.
\end{abstract}

\section{Introduction}

Nasopharyngeal carcinoma (NPC) is rare in most populations, although its incidence is particularly high among Asians,

Correspondence to: Dr Yan Liu, The Fifth Department of Chemotherapy, Affiliated Tumor Hospital of Guangxi Medical University, Nanning 530021, P.R. China

E-mail:360441575@qq.com

${ }^{*}$ Contributed equally

Key words: autophagy, nasopharyngeal carcinoma, radiosensitivity, autophagy related 5, $\operatorname{Rad} 51$ particularly in Southern China with an age standardized incidence rate of $\sim 25$ cases per 100,000 individuals per year (1). As a traditional treatment modality, radiotherapy remains the mainstay of treatment for NPC due to its radiosensitivity, even though it has been applied for cancer therapy for more than a century. If diagnosed and treated at an early stage, most NPC patients can be cured. However, locoregional recurrence or distant metastasis may occur in some patients after primary radiotherapy or chemo-irradiation due to the radiation resistance of cancer cells (2). Due to the presence of tumor cell heterogeneity, cancer cells may exhibit different degrees of radiosensitivity even when they are of the same histological differentiation status, which may contribute to the poor overall survival of NPC patients after recurrence. It follows, therefore, that the development of novel therapeutic strategies to overcome radioresistance and enhance radiosensitivity of NPC are urgently needed.

In regards to the primary mechanism of radiation-induced cell death, apoptosis is not the predominant form of cell death, accounting for only $20 \%$ of death (3). Another cell death pathway, namely autophagy, has emerged as a crucial mechanism of tumor cell death induced by radiation (4). Autophagy, a multi-step process that involves degradation of long-lived cellular proteins and organelles, is a genetically programmed, highly conserved process that occurs in eukaryotes from yeast to mammals. Recently, studies have shown that autophagy plays a vital role in protecting cells against adverse conditions (5-7), including irradiation (8). Inhibition of autophagy plays an active role in radiosensitization in several cancer cell types (9) as well as in NPC cells (10). Although many studies have rigorously attempted to elucidate the mechanism of autophagy in cancer treatments, it remains unclear.

DNA double-strand breaks (DSBs) are the most critical event in ionizing radiation (IR)-induced cell death and can be efficiently repaired by DNA homologous recombination, which is essential for maintaining genomic stability after IR, while Rad51 is a key protein of homologous recombination in resynthesizing the damaged region of the DNA (11). Overexpression of Rad51 may be a possible mechanism with which to suppress recombination defects and increase the resistance of mammalian cells to IR (12), suggesting that Rad51 expression may play an essential role in radioresistance. 
Previous research has demonstrated that homologous recombination may mediate cellular resistance to radiation therapy (13), and inhibition of autophagy contributes to the radiosensitivity of CNE-2 cells (14). Therefore, we hypothesized that inhibition of autophagy could enhance the radiosensitivity of NPC cells by influencing the DNA homologous recombination system. To test this hypothesis, we investigated the effect of autophagy inhibition on the radiosensitivity of NPC cells and elucidated the role of Rad51 in the regulation of radiosensitivity.

\section{Materials and methods}

Cell culture and irradiation conditions. The human NPC cell lines, CNE-1 and CNE-2, were obtained from the Chinese Academy of Sciences Cell Bank and were cultured in Roswell Park Memorial Institute (RPMI)-1640 medium supplemented with $10 \%$ fetal bovine serum, $100 \mathrm{U} / \mathrm{ml}$ of penicillin and $100 \mu \mathrm{g} / \mathrm{ml}$ of streptomycin (all from Invitrogen, Carlsbad, CA, USA). Cultures were maintained in a humidified atmosphere of $5 \% \mathrm{CO}_{2}$ at $37^{\circ} \mathrm{C}$. 3-Methyladenine (3-MA) and chloroquine (CQ) were obtained from Sigma-Aldrich (Shanghai, China). RI-1 was purchased from ChemBridge Corp. (San Diego, CA, USA). BO2 was purchased from Chief-East Tech Co., Ltd. (Beijing, China). All irradiations were delivered using 6-MV $\mathrm{X}$-rays with a linear accelerator (Elekta, Sweden) with a dose rate of $220 \mathrm{cGy} / \mathrm{min}$; SSD, $100 \mathrm{~cm}$.

shRNA. A scrambled hairpin (SCR) was used as a negative control (Invitrogen), and Stealth RNAi ${ }^{\mathrm{TM}}$ shRNA duplex oligoribonucleotides targeting human Atg5 were obtained from Invitrogen. The sequence of SCR-shRNA was CCT ACG CCA CCA ATT TCG T; Atg5-1-shRNA was ATT GGC TCA ATT CCA TGA ATC and Atg5-2-shRNA was AAG CAA ATA GTA TGG TTC TGC. The shRNA was transfected into CNE1 and CNE2 cells using shRNA transfection reagent (Santa Cruz Biotechnology, Inc., Santa Cruz, CA, USA) according to the manufacturer's protocol.

Cell viability assay. The measurement of the viable cell mass was assessed by the Cell Counting Kit-8 (Dojin Laboratories, Kumamoto, Japan), as previously described (15).

Real-time PCR assay. Cells were collected to extract the total cellular mRNA using TRIzol reagent (Invitrogen). cDNA was synthesized using Moloney murine leukemia virus reverse transcriptase (Promega, Madison, WI, USA) and $2 \mu \mathrm{g}$ of total RNA and oligo(dT) $)_{18}$ primers. Two-microliter aliquots of cDNA were used for PCR amplification. Real-time PCR was performed in triplicate using the SYBR PrimeScript RT-PCR kit (Takara, Dalian, China). The sequences of the Atg5 primers listed were TGG ATT TCG TTA TAT CC CCT TT AG (sense) and CCT AGT GTG TGC AAC TGT CCA (antisense); the sequences of the Rad51 primers listed were 5'-TGG CCC ACA ACC CAT TTC AC-3' (sense) and 5'-TCA ATG TAC ATG GCC TTT CCT TCA C-3' (antisense). Total sample RNA was normalized to endogenous human glyceraldehyde-3-phosphate dehydrogenase (GAPDH) mRNA. Thermocycler conditions included an initial hold at $50^{\circ} \mathrm{C}$ for $2 \mathrm{~min}$ and then $95^{\circ} \mathrm{C}$ for $10 \mathrm{~min}$; this was followed by a two-step PCR program of $95^{\circ} \mathrm{C}$ for $15 \mathrm{sec}$ and $60^{\circ} \mathrm{C}$ for $60 \mathrm{sec}$ repeated for 40 cycles on an Mx4000 system (Stratagene, La Jolla, CA, USA), on which data were collected and quantitatively analyzed. The expression level of mRNA was presented as fold-change relative to an untreated control.

Western blot analysis. Cells were washed in PBS solution, and the protein was extracted according to an established protocol. Nuclear extract proteins were quantified using the Bio-Rad protein assay. Proteins were then mixed with Laemmli sample buffer, heated at $65^{\circ} \mathrm{C}$ for $10 \mathrm{~min}$, loaded ( $\left.20 \mu \mathrm{g} / \mathrm{sample}\right)$, separated by SDS-polyacrylamide gel (7.5\%) electrophoresis under denaturing conditions and electroblotted on nitrocellulose membranes. The nitrocellulose membranes were blocked by incubation in blocking buffer (1\% BSA in Tris-buffered saline, $0.1 \%$ Tween-20), incubated with the anti-Atg5 antibody (1:500 polyclonal; Bethyl), washed and incubated with the anti-rabbit peroxidase-conjugated secondary antibody (1:10,000; Sigma). Signals were visualized by chemiluminescent detection. Blots were quantified using Quantity One software (Bio-Rad), and Atg5 (Epitomics, Inc., USA) expression (peak intensity) was normalized to values in the control group. Equal loading of samples was verified by Coomassie blue staining of simultaneously run gels. Gels were run four times, and the images shown are representative.

Cell cycle analysis by flow cytometry. Approximately $1 \times 10^{6}$ cells were collected and fixed overnight in $70 \%$ ethanol at $4^{\circ} \mathrm{C}$. Cells were then washed in PBS and stained with PI (Sigma) in the presence of DNase-free RNase. After a 30-min incubation at room temperature in the dark, cells were filtered through a $40-\mu \mathrm{m}$ diameter mesh to remove clumps of nuclei, and cells within the cell cycle compartments (sub-G1, S or G2-M) were determined via a flow cytometer using CellQuest software (BD FACSAria, Becton-Dickinson), acquiring 10,000 events. The percentages of sub-G1 nuclei in the population were determined as apoptosis.

Cell apoptosis assay. Cells ( $2 \times 10^{5}$ per well) were cultured in 6-well plates to 70-80\% confluency. Annexin V-FITC assay was used to measure apoptotic cells by flow cytometry according to the manufacturer's instructions (Nanjing KeyGen Biotech Co., Ltd., Nanjing, China; cat. KGA108). Briefly, cells were collected by trypsinization, washed with ice-cold phosphate-buffered saline (PBS) twice and resuspended in $300 \mu \mathrm{l} 1 \mathrm{X}$ binding buffer containing $5 \mu \mathrm{l}$ Annexin $\mathrm{V}$ and $5 \mu \mathrm{l}$ PI for $30 \mathrm{~min}$ at room temperature in the dark. After incubation, at least 10,000 cells were measured on a BD FACSAria flow cytometer. Results are expressed as the percentage of apoptotic cells at early stage (PI-negative and Annexin V-positive). Meanwhile, apoptosis was detected by staining cells with DAPI based on the nuclear morphology, identifying those cells with condensed and fragmented nuclei as apoptotic.

Statistical analysis. All of the experiments were repeated at least three times. The data are expressed as means \pm SD. Statistical analysis was performed using the Student's t-test (two-tailed). The criterion for statistical significance was determined to be $\mathrm{P}<0.05$. 
A

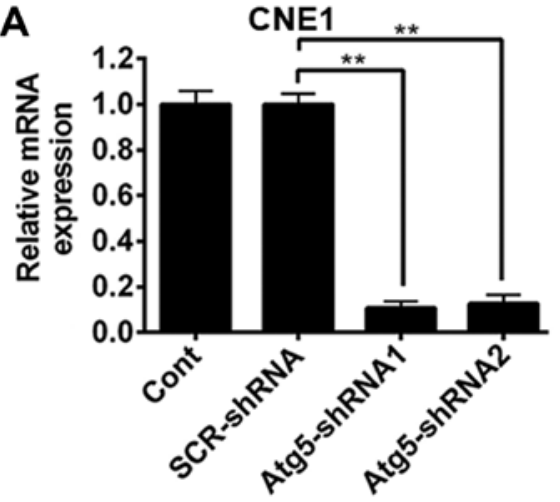

C

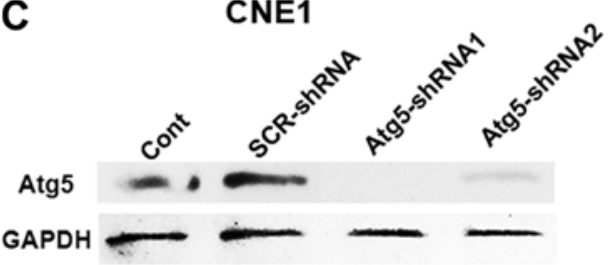

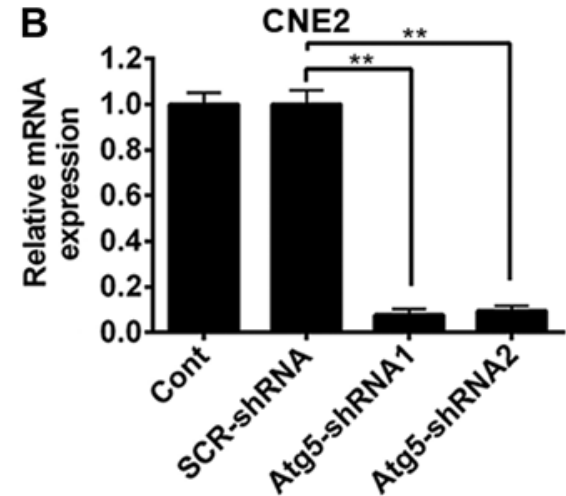

D

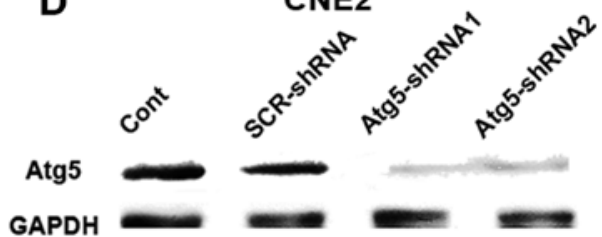

Figure 1. Lentivirus-delivered shRNA stably decreases Atg5 expression in human nasopharyngeal carcinoma cells. (A-D) CNE1 and CNE2 cells were infected by a lentivirus delivered shRNA. Total RNA and protein were extracted 5 days after infection. (A and B) Relative Atg5 mRNA expression was detected by qRT-PCR. Data are the mean \pm SD $\left(n=3 ;{ }^{* *} \mathrm{P}<0.01\right)$. (C and D) Atg5 protein level was determined by western blot analysis.

\section{Results}

Suppression of Atg5 enhances the radiosensitivity of human NPC cells. To investigate the function of the autophagy related 5 (Atg5) gene in NPC cells after radiation, specific lentivirus-delivered shRNA to the Atg5 gene was used to induce Atg5 gene silencing by a mechanism that involves the RNA-induced silencing complex (RISC). Transduced cells were expanded, and Atg5 mRNA expression was detected by RT-qPCR while the Atg5 protein level was determined by western blot analysis 5 days after infection. As shown in Fig. 1A, silencing of the Atg5 gene markedly decreased the expression of Atg5 mRNA in the CNE-1 cells either transfected with Atg5-targeted shRNA1 or Atg5-targeted shRNA2 compared with the non-targeted shRNA CNE-1 cells and control cells. Similarly, transduction of CNE1 cells with the Atg5-shRNA1 and Atg5-shRNA2 construct virus resulted in nearly complete elimination of Atg 5 protein expression (Fig. 1C). In contrast, the levels of Atg5 protein in cells transduced with the lentiviral vector including the non-specific construct shRNA sequence and control cells did not exhibit an obvious change when compared with GAPDH. Similar results were observed in the CNE-2 cells (Fig. 1B and D). The results above suggest that silencing of the Atg5 gene reduced the autophagy of NPC cells.

It has been demonstrated that cells die mainly via two pathways, necrosis and apoptosis (16). Ionizing radiation (IR) kills cancer cells mainly through the induction of DNA doublestrand breaks (DSBs), apoptosis as well as autophagy, whereas autophagy is widely considered to be a protective mechanism against IR. Thus, we hypothesized that autophagy may protect NPC cells from IR-induced cell death by reducing apoptosis and DNA damage. To test this, we further investigated the effect of Atg5 silencing on IR-induced cell apoptosis and DNA damage. Forty-eight hours after 4-Gy X-ray irradiation treatment, cell viability was evaluated by the CCK- 8 assay. As shown in Fig. 2A and B, following the silencing of the Atg5 gene, the cell viability of CNE-1 and CNE-2 cells significantly decreased compared with that of the scrambled and control cells.

The decrease in the viability of the cells transfected with Atg5-targeted shRNA indicated an increase in cell death of NPC cells after radiation. Apoptosis, known as type I programmed cell death, could underlie the biological function of radiation. We investigated whether there was an enhancement of cell apoptosis induced by radiation after Atg5 silencing. To evaluate apoptosis, the percentage of apoptotic cells was assessed by flow cytometry with Annexin V/PI double staining analysis. Results showed that CNE-1 cells transfected with the Atg5-targeted shRNA had a higher proportion of apoptotic cells (Fig. 2C) compared to that of cells transfected with the non-targeted shRNA and control cells. Similar data were obtained using CNE-2 cells (Fig. 2D).

The formation of $\gamma-\mathrm{H} 2 \mathrm{AX}$, namely phosphorylation of a histone protein $\mathrm{H} 2 \mathrm{AX}$, indicates the early response of cells to IR-induced DNA damage (17). $\gamma$-H2AX is commonly accepted as a biomarker that indicates the presence of a DSB. Hence, we detected the protein level of $\gamma-\mathrm{H} 2 \mathrm{AX}$ to confirm the DNA damage after radiation in NPC cells. Western blot analysis showed that the protein level of $\gamma$-H2AX in the CNE1 and CNE2 cells was increased in all experimental groups after IR (Fig. 2E and F). However, a higher increase in the protein level of $\gamma-\mathrm{H} 2 \mathrm{AX}$ was observed as the cells were pre-interfered by Atg5-targeted shRNA. Together, the results suggest that suppression of Atg5, to some extent, enhanced the susceptibility of NPC cells to radiation.

Inhibition of autophagy decreases the expression of Rad51. The above data confirmed that suppression of Atg5 increased the susceptibility of NPC cells to radiation, while the mechanism remains unclear. Radiation induces $\mathrm{G} 2 / \mathrm{M}$ phase arrest in 
A

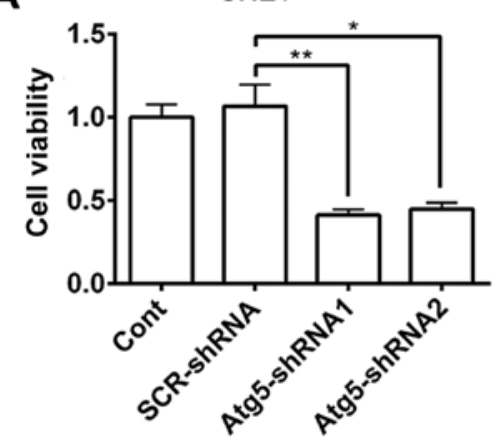

C

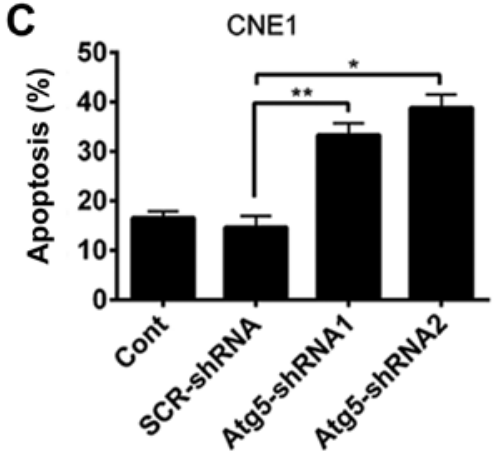

E

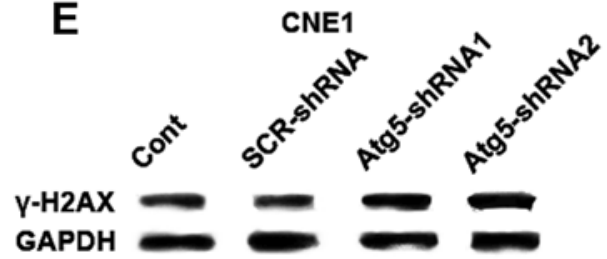

B

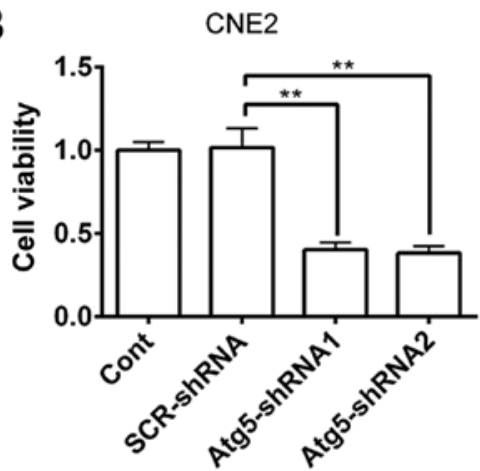

D

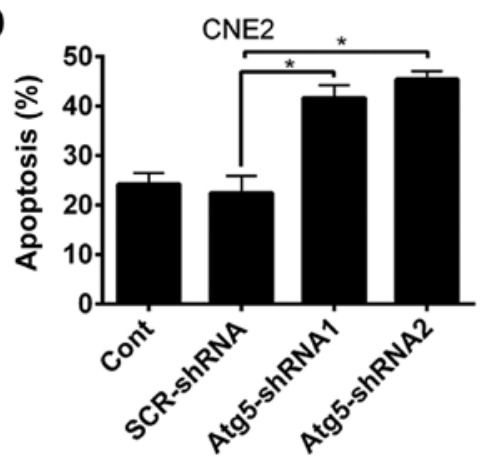

$\mathbf{F}$

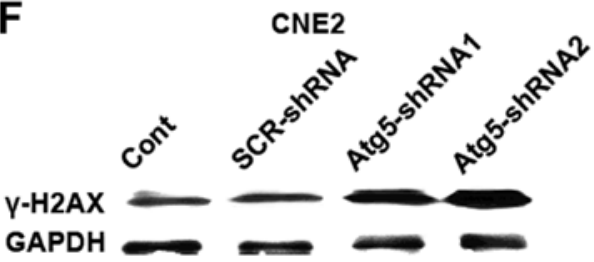

Figure 2. Suppression of Atg5 aggravates IR-induced DNA damage and apoptosis in human nasopharyngeal carcinoma cells. (A-F) CNE1 and CNE2 cells were treated with 4-Gy irradiation. After $48 \mathrm{~h}$, (A and B) cell viability was evaluated by the CCK-8 assay, (C and D) the percentage of apoptotic cells was detected by flow cytometry with Annexin V/PI double staining and (E and F) the protein level of $\gamma$-H2AX was determined by western blot analysis. (A-D) Data are the mean $\pm \mathrm{SD}\left(\mathrm{n}=3 ;{ }^{*} \mathrm{P}<0.05 ;{ }^{* *} \mathrm{P}<0.01\right)$. IR, ionizing radiation.

various types of cancer cells $(18,19)$. This results in enhanced radiosensitivity of cancer cells for the reason that cells are most radiosensitive in the $\mathrm{M}$ and $\mathrm{G} 2$ phases. Therefore, we aimed to ascertain whether the radiosensitization effect of Atg5 silencing was due to $\mathrm{G} 2 / \mathrm{M}$ phase arrest. Contrary to our expectation, cell cycle analysis by flow cytometric measurement revealed that suppression of Atg5 led to cell cycle arrest in the G1 phase where cells are less radiosensitive, while the percentage of cells in the $\mathrm{G} 2 / \mathrm{M}$ phase was decreased dramatically in the CNE-2 cells; both results achieving statistical significance (Fig. 3B). However, no difference in cell cycle distribution was noted in the CNE-1 cells (Fig. 3A).

Radiation-induced cell damage mainly results in DNA DSBs (20). Rad51, a key protein of homologous recombination, has been demonstrated to play a critical role in the repair of DNA DSBs. We aimed to ascertain whether Rad51 is related to the radiosensitization effect following silencing of the Atg5 gene. Thus, expression of Rad51 mRNA in NPC cells was analyzed. The RT-qPCR analysis revealed that the expression of Rad51 mRNA demonstrated a notable rise in the negative control and mock-transfected control cells when treated with radiation. However, when cells were transfected with the Atg5-targeted shRNA, which was proven to inhibit autophagy in the NPC cells, the Rad51 mRNA expression revealed a significant decline both in cells treated with or without radiation when compared with that of the negative control and mock-transfected control cells. Moreover, a greater decrease in the expression of Rad51 mRNA was observed as the cells were subjected to a combined treated with radiation (Fig. 4A and B).

To further confirm the effect of autophagy inhibition on Rad51 mRNA expression, the control cells were treated with 3-MA or CQ alone or a combined treatment with irradiation followed by Rad51 mRNA expression analysis with RT-qPCR. Fig. 4C and D shows that the Rad51 mRNA expression levels exhibited a completely positive response which were significantly upregulated when IR was administered to the control cells; however, when the cells were treated with 3-MA or CQ, the expression of Rad51 mRNA showed a marked decline compared to that of the untreated cells. Moreover, the disparity became more marked when cells received IR. Notably, IR barely altered the expression of Rad51 mRNA as long as the cells were treated with 3-MA or CQ. As expected, the effect of autophagy inhibition on the expression of Rad51 was ascertained by $3-\mathrm{MA}$ and $\mathrm{CQ}$, indicating that autophagy inhibition may suppress the expression of Rad51. 

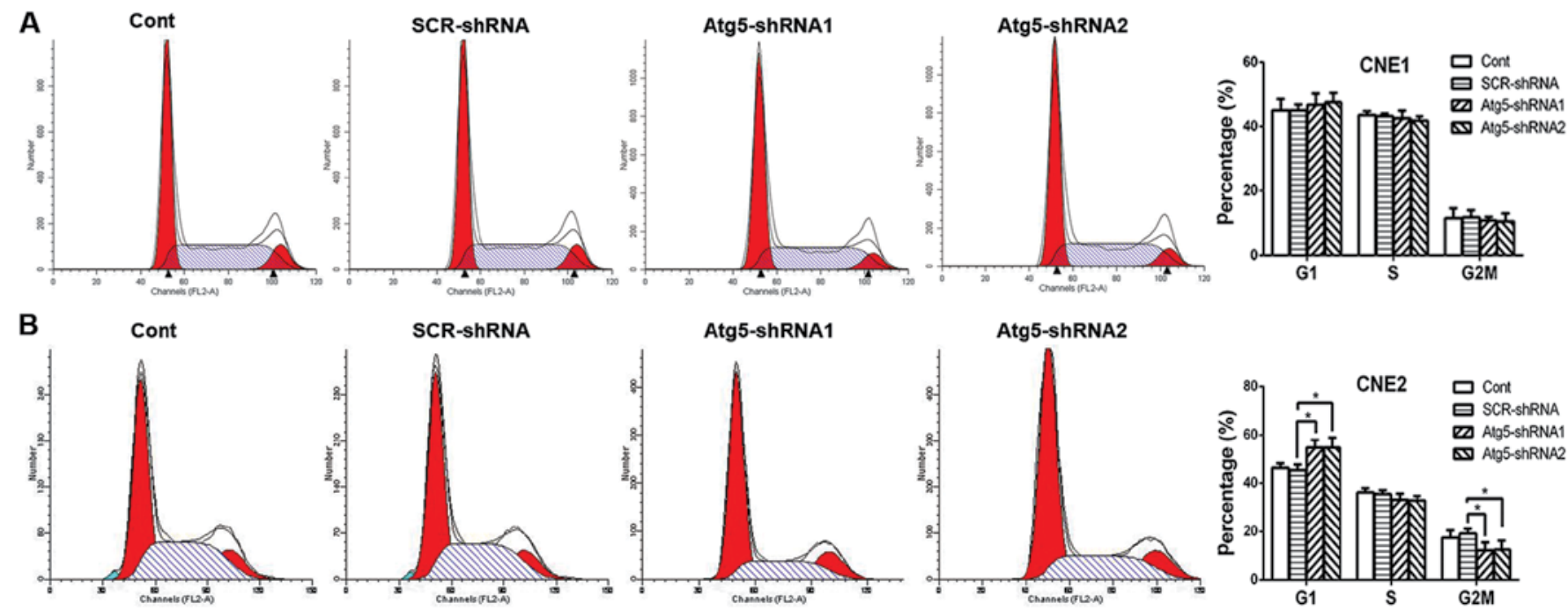

Figure 3. Suppression of Atg5 does not accelerated cell cycle progression in human nasopharyngeal carcinoma cells. (A and B) Cell cycle analysis was conducted using PI staining and FACS analysis in (A) CNE1 and (B) CNE2 cells. (Right panels) The percentages of cells in each cell cycle phase are expressed as the mean $\pm \mathrm{SD}\left(\mathrm{n}=3 ;{ }^{*} \mathrm{P}<0.05\right)$.
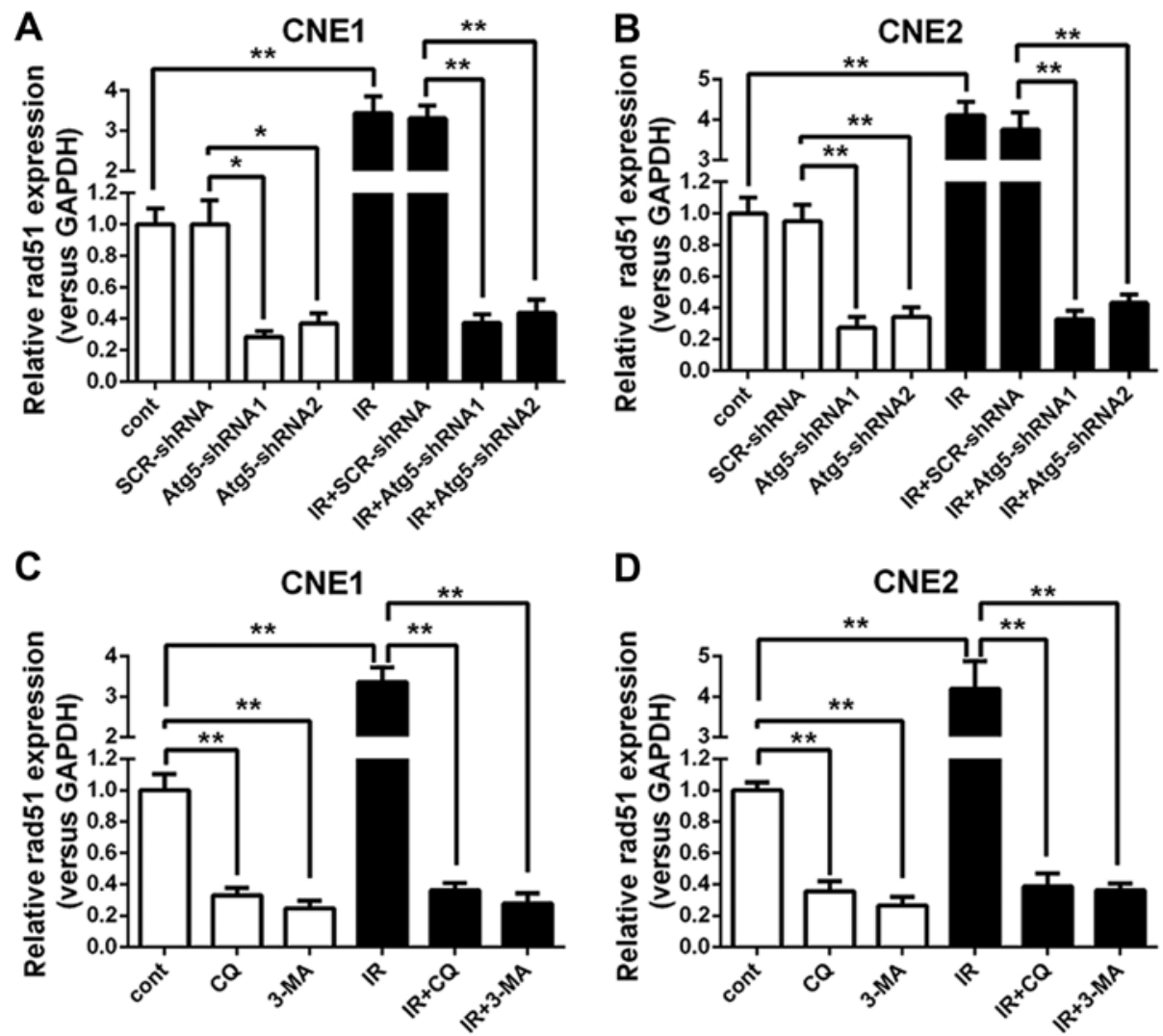

Figure 4. Inhibition of autophagy suppresses Rad51 mRNA expression in human nasopharyngeal carcinoma cells. (A-D) CNE1 and CNE2 cells were treated or not treated with 4-Gy irradiation. Two hours after irradiation, total RNA was extracted and the relative Rad51 mRNA expression was detected by qRT-PCR. Data are the mean $\pm \operatorname{SEM}\left(n=3 ;{ }^{*} \mathrm{P}<0.05 ;{ }^{* *} \mathrm{P}<0.01\right)$. (A and B) The cells were stably infected by lentivirus delivered shRNA before irradiation. (C and $\left.D\right)$ The cells were pretreated with the autophagy inhibitor CQ $(10 \mu \mathrm{M})$ or $3-\mathrm{MA}(5 \mathrm{mM}) 2 \mathrm{~h}$ before irradiation.

Inhibition of autophagy enhances the sensitivity of NPC cells to IR by reducing Rad51 expression. Prior studies have demonstrated that B02 is a specific inhibitor of human Rad51 (21), while RI-1 acts as a chemical inhibitor of Rad51 that disrupts homologous recombination in human cells (22). Thus, to demonstrate the function of $\operatorname{Rad} 51$ in the radiosensitivity of NPC cells, we examined the influence of Rad51 suppression on radiation sensitivity by treating NPC cells with B02 or RI-1 $1 \mathrm{~h}$ before the treatment of 4-Gy irradiation. After $48 \mathrm{~h}$, cell viability was evaluated using the CCK- 8 assay. As shown in 

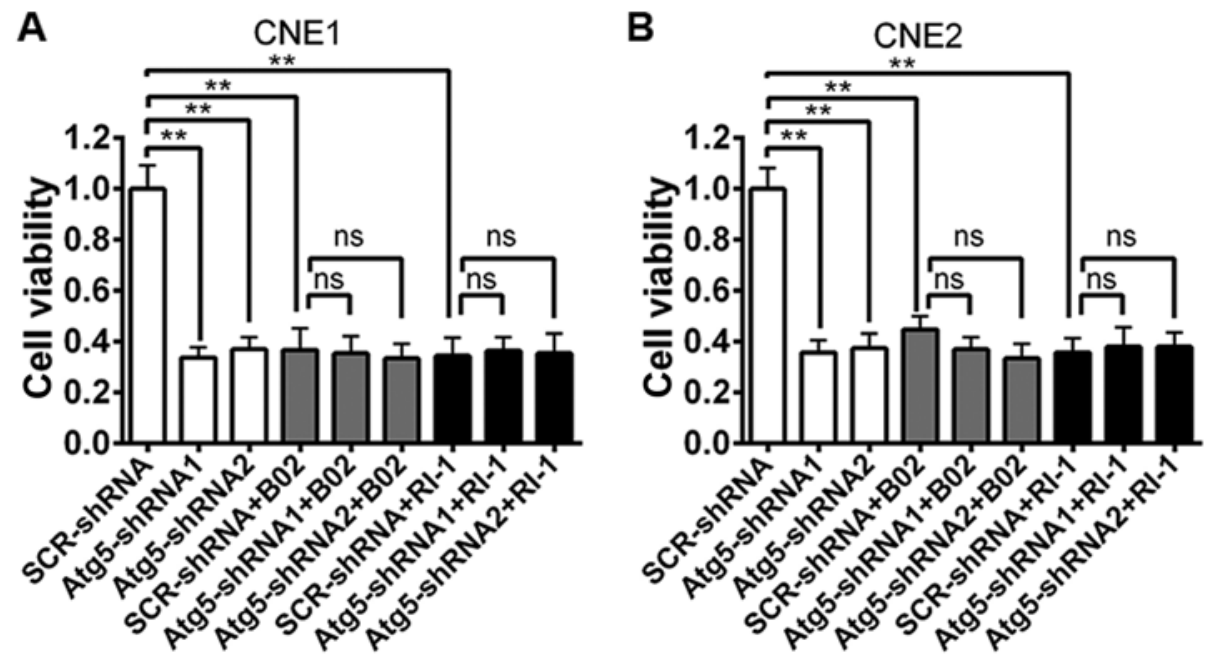

Figure 5. Suppression of Atg5 has no impact on the radiosensitivity of Rad51 inhibitor-treated human nasopharyngeal carcinoma cells. (A and B) CNE1 and CNE2 cells were pre-treated by Rad51 inhibitor B02 $(25 \mu \mathrm{M})$ or RI-1 $(25 \mu \mathrm{M}) 1 \mathrm{~h}$ before the treatment with 4-Gy irradiation. After $48 \mathrm{~h}$, cell viability was evaluated by the CCK- 8 assay. Data are the mean $\pm \mathrm{SD}\left(\mathrm{n}=3 ;{ }^{* *} \mathrm{P}<0.01 ; \mathrm{ns}\right.$, not significant).
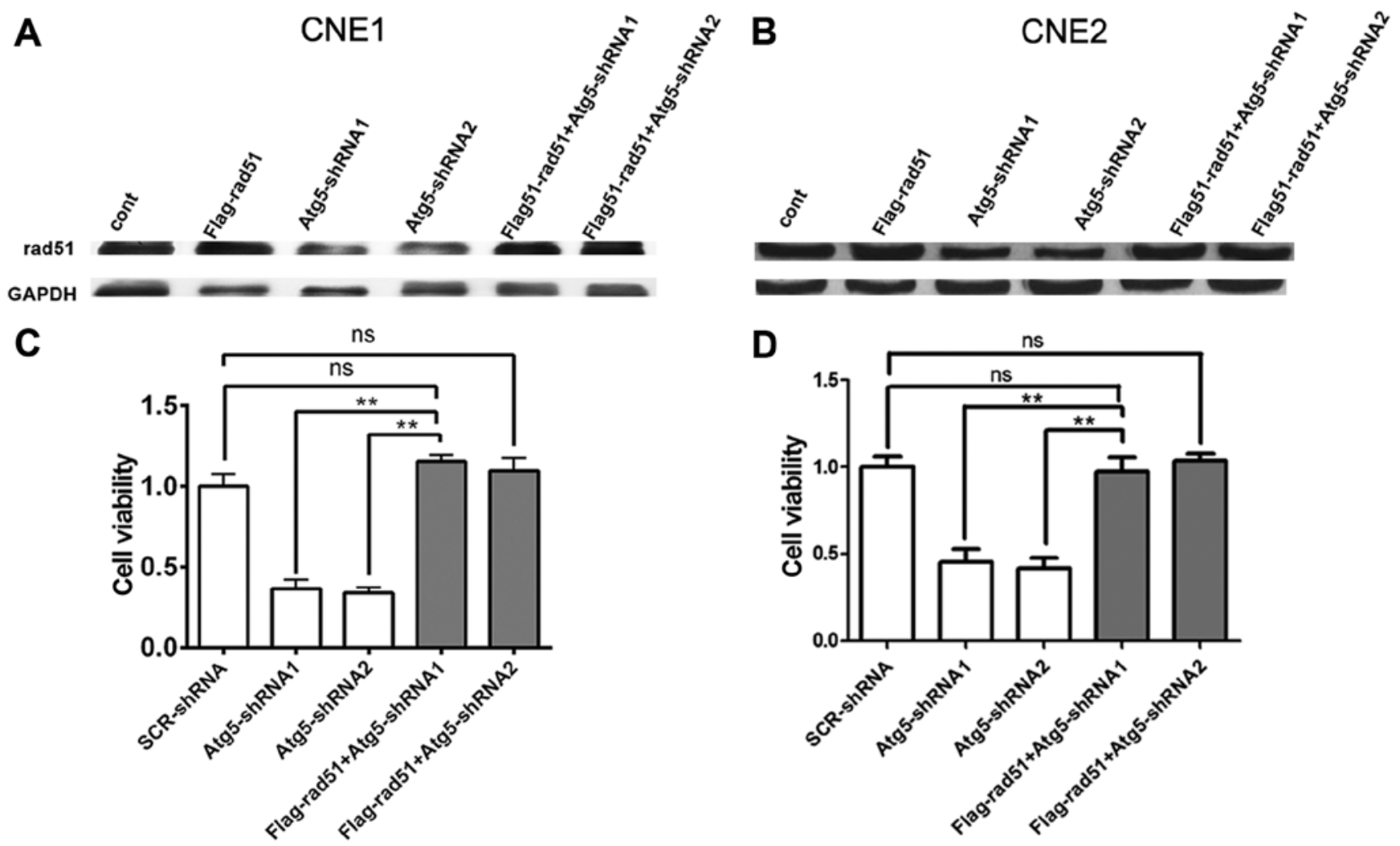

D

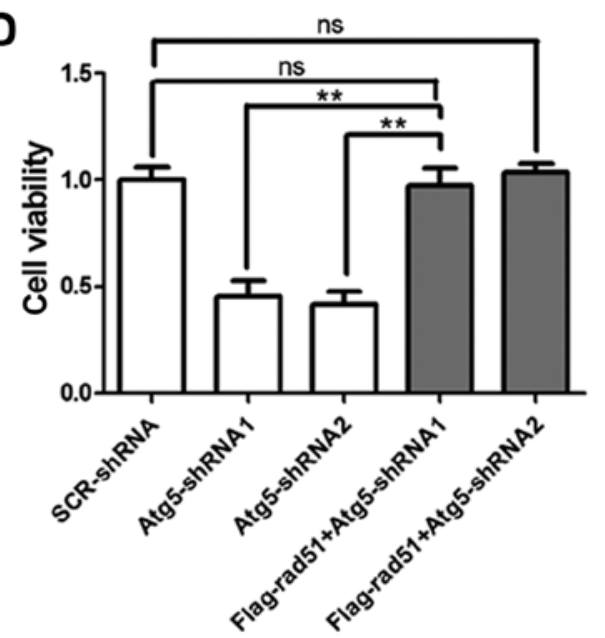

Figure 6. Overexpression of Rad51 reverses the enhanced radiosensitivity by Atg5 suppression in human nasopharyngeal carcinoma cells. (A and B) CNE1 and CNE2 cells were transfected with the Flag-Rad51 vector. After $48 \mathrm{~h}$, the total protein was extracted and the Rad51 protein level was determined by western blot analysis. (C and D) Forty-eight hours after transfected with the Flag-Rad51 vector, the cells were treated with 4-Gy irradiation. After the next $48 \mathrm{~h}$, cell viability was evaluated by the CCK- 8 assay. Data are the mean $\pm \mathrm{SD}\left(\mathrm{n}=3 ;{ }^{* * *} \mathrm{P}<0.01 ; \mathrm{ns}\right.$, not significant).

Fig. 5, a marked decrease in cell viability was observed both in cells transfected with the Atg5-targeted shRNA alone or scrambled cells treated with the Rad51 inhibitor when compared to the cell viability of the scrambled cells. However, no change was noted between the cells transfected with the Atg5-targeted shRNA and the non-targeted shRNA when cells were pre-treated with B02 or RI-1.
To further validate the relationship between Rad51 and the radioresistance of NPC cells, CNE1 and CNE2 cells were transfected with the Flag-Rad51 vector. After 48 h, the total protein level of Rad51 was determined by western blot analysis. After the next $48 \mathrm{~h}$ when cells were treated by 4-Gy irradiation, cell viability was evaluated by the CCK-8 assay. Western blot analysis confirmed Rad51 overexpression 
in cells transfected with the Flag-Rad51 vector and the control cells when compared to that of cells transfected with the Atg5-targeted shRNA after irradiation. However, the low expression of Rad51 in cells transfected with the Atg5targeted shRNA exhibited a reverse upregulation when cells were transfected with the Flag-Rad51 vector (Fig. 6A and B). Cell viability assays revealed that the viability of the cells transfected with the Atg5-targeted shRNA was significantly decreased compared to that of the mock-transfected control cells, but showed an increase when cells were transfected with the Flag-Rad51 vector before irradiation, which reversed the enhanced radiosensitivity by Atg5 suppression in the CNE1 and CNE2 cells (Fig. 6C and D). Taken together, the above data indicate a correlation between a reduced Rad51 protein level and increased radiosensitivity to radiation. Thus, the level of Rad51 protein is inversely correlated with sensitivity to radiation in NPC cells.

\section{Discussion}

Autophagy, or 'self-eating', is a double-edged sword that can both suppress cancer initiation and promote the growth of established cancers. A large body of literature has strived to elucidate the role of autophagy in cancer cells in response to cancer therapy. However, whether autophagy contributes to cell death or rather represents a survival mechanism remains controversial $(23,24)$. White revealed that suppression or deficiency of autophagy genes are associated with diseases, including cancer (25). Anticancer agents, such as tamoxifen (26), rapamycin (27), temozolomide (28) and IR (29), have been reported to induce autophagy. After exposure to IR, autophagy can frequently be observed in cancer cells, and radiosensitivity can be increased by inhibition of autophagy (30). Consistent with this, in the present study, we showed that inhibition of autophagy by knockdown of Atg5 (Fig. 1) enhanced the cytotoxicity of radiotherapy in CNE-1 or CNE-2 cells, as determined from a markedly decrease in cell viability and a higher proportion of apoptotic cells (Fig. 2). Our findings were further strengthened by a recent study suggesting that blockade of autophagy by transduction of specific target siRNAs led to downregulation of the autophagy-related genes, beclin 1, atg3, atg4b, atg 4c, atg5 and atg12 in cell lines, resulting in enhanced cytotoxicity of radiotherapy in cancer cells. However, its regulatory mechanisms remain elusive.

It has been demonstrated that the factors that influence intrinsic radiosensitivity of cell subpopulations include the level of hypoxia, DNA repair capacity and cell cycle phase. Among these, regulation of the cell cycle may be the most important determinant of IR sensitivity (31). Cells are most radiosensitive in the $M$ and $G 2$ phases, less sensitive in the G1 phase and most radioresistant in the $S$ phase (31).

However, we found that the enhanced cytotoxicity of radiotherapy in $\mathrm{CNE}-1$ or $\mathrm{CNE}-2$ cells did not involve cell cycle arrest. Suppression of Atg5 only resulted in G1 phase arrest while the percentage of cells in the G2/M phase was significantly reduced in the CNE-2 cells following radiation, yet no difference in cell cycle distribution in CNE-1 cells was detected (Fig. 3). Explanation of this may include the short time of irradiation for only $48 \mathrm{~h}$ in our experiment. This may not have been long enough to induce significant cell cycle blockage and the cell type and the radiation dose used must be taken into consideration. Therefore, in the present study, cell cycle arrest was not the main mechanism of radiosensitivity in NPC cells.

It has been reported that ionizing radiation mainly results in the induction of DNA DSBs if left unrepaired (32), while Rad51 has been demonstrated to be the central player in the initiation of homologous recombination that plays a critical role in the repair of DNA DSBs. Rad51 is not only involved in the progression of carcinogenesis but also in the resistance to anticancer treatments (33). Enhanced Rad51 protein expression can influence the chemoradiotherapy treatment outcome as well as potentiate radioresistance in tumor cells (34). Further investigation by us indicated that inhibition of autophagy with Atg5-targeted shRNA and an autophagy inhibitor both resulted in decreased expression of Rad51, yet exhibited no change even when cells were treated with IR (Fig. 4). These data indicate that inhibition of autophagy impairs the survival mechanism of NPC cells after radiation by reducing Rad51 expression. Thus, we hypothesized that suppression of Rad51 expression rather than cell cycle blockage is a possible mechanism for autophagy-inhibited induced radiosensitization in NPC cells.

For characterizing the role of Rad51, CNE1 and CNE2 cells were treated with RI-1or B02 prior to irradiation. Regardless of whether cells were pre-treated with the Rad51 inhibitor or subjected to knockdown of Atg5, the cell viability was markedly decreased when compared to the scrambled cells without treatment by the Rad51 inhibitor. Moreover, the extent of cell viability was decreased between cells transfected with the Atg5-targeted shRNA and the scrambled cells and was not meaningful by statistical analysis when cells were pre-treated with B02 or RI-1 (Fig. 5A and B). These results indicate that the target by which the inhibition of autophagy mediates the radioresistance of NPC cells may be the same as that of B02 and RI-1. Furthermore, the upregulation of Rad51 protein by transfecting the Flag-Rad51 vector into CNE-1 and CNE-2 cells reversed the enhanced radiosensitivity by Atg5 suppression with increased cell viability to radiation (Fig. 6C and D). Corresponding to our results, previous reseach concluded that overexpression of Rad51 contributes to the resistance of IR and other DNA-damaging agents (12), while downregulation of Rad51 protein may sensitize tumor cells to IR (35). These results indicate that Rad51 may not only be involved in the resistance to radiation which results in local residue but also in the locoregional recurrence or distant metastasis of NPC. Nevertheless, the interaction between Rad51 and autophagy in the radiation therapy of NPC is complex and further studies are warranted to support the conclusion.

In summary, our study provides compelling data supporting our hypothesis that inhibition of autophagy enhances the radiosensitivity of NPC cells by reducing Rad51 expression. Thus, the autophagic process of NPC cells is a self-protective mechanism against radiation. Rad51 targeted therapy may be investigated as a potential novel agent for the adjuvant treatment of traditional radiation of NPC, particularly to ascertain whether their combination and maintenance treatment of Rad51 targeted therapy following radiation can enhance the therapeutic effect of radiation, in order to minimize locoregional recurrence or distant metastasis and maximize the outcome of NPC. 


\section{Acknowledgements}

The study was supported by the Guangxi Medical University Fund for Young Scientists (GXMUYSF11), the Guangxi Natural Science Foundation (2013GXNSFAA019236) and the Guangxi Natural Science Foundation for Young Scientists (2014GXNSFBA118141).

\section{References}

1. Chang ET and Adami HO: The enigmatic epidemiology of nasopharyngeal carcinoma. Cancer Epidemiol Biomarkers Prev 15: 1765-1777, 2006.

2. Hou H, Li D, Cheng D, Li L, Liu Y and Zhou Y: Cellular redox status regulates emodin-induced radiosensitization of nasopharyngeal carcinoma cells in vitro and in vivo. J Pharmaceutics 2013: 2013.

3. Verheij M and Bartelink H: Radiation-induced apoptosis. Cell Tissue Res 301: 133-142, 2000.

4. Zois CE and Koukourakis MI: Radiation-induced autophagy in normal and cancer cells: towards novel cytoprotection and radio-sensitization policies? Autophagy 5: 442-450, 2009.

5. Liu WM, Huang P, Kar N, et al: Lyn facilitates glioblastoma cell survival under conditions of nutrient deprivation by promoting autophagy. PloS One 8: e70804, 2013.

6. Oehme I, Linke JP, Böck BC, et al: Histone deacetylase 10 promotes autophagy-mediated cell survival. Proc Natl Acad Sci USA 110: E2592-E2601, 2013.

7. Selvakumaran M, Amaravadi RK, Vasilevskaya IA and O'Dwyer PJ: Autophagy inhibition sensitizes colon cancer cells to antiangiogenic and cytotoxic therapy. Clin Cancer Res 19: 2995-3007, 2013.

8. Lomonaco SL, Finniss S, Xiang C, et al: The induction of autophagy by $\gamma$-radiation contributes to the radioresistance of glioma stem cells. Int J Cancer 125: 717-722, 2009.

9. Apel A, Herr I, Schwarz H, Rodemann HP and Mayer A: Blocked autophagy sensitizes resistant carcinoma cells to radiation therapy. Cancer Res 68: 1485-1494, 2008.

10. Wang Y, Yin W and Zhu X: Blocked autophagy enhances radiosensitivity of nasopharyngeal carcinoma cell line CNE-2 in vitro. Acta Otolaryngol 134: 105-110, 2014.

11. Mazin AV and Mazina OM: RAD51 is a key protein of DNA repair and homologous recombination in humans. 281-302, 2013.

12. Vispé S, Cazaux C, Lesca C and Defais M: Overexpression of Rad51 protein stimulates homologous recombination and increases resistance of mammalian cells to ionizing radiation. Nucleic Acids Res 26: 2859-2864, 1998.

13. Somaiah N, Yarnold J, Lagerqvist A, Rothkamm K and Helleday T: Homologous recombination mediates cellular resistance and fraction size sensitivity to radiation therapy. Radiother Oncol 108: 155-161, 2013.

14. Zhou ZR, Zhu XD, Zhao W, et al: Poly(ADP-ribose) polymerase-1 regulates the mechanism of irradiation-induced CNE-2 human nasopharyngeal carcinoma cell autophagy and inhibition of autophagy contributes to the radiation sensitization of CNE-2 cells. Oncol Rep 29: 2498-2506, 2013.

15. Takasu H, Sugita A, Uchiyama Y, et al: c-Fos protein as a target of anti-osteoclastogenic action of vitamin D, and synthesis of new analogs. J Clin Invest 116: 528-535, 2006.

16. Menna-Barreto RF, Salomão K, Dantas AP, et al: Different cell death pathways induced by drugs in Trypanosoma cruzi: an ultrastructural study. Micron 40: 157-168, 2009.
17. Dickey JS, Redon CE, Nakamura AJ, Baird BJ, Sedelnikova OA and Bonner WM: H2AX: functional roles and potential applications. Chromosoma 118: 683-692, 2009.

18. Bae Y, Jung SH, Kim GY, Rhim H and Kang S: Hip2 ubiquitinconjugating enzyme overcomes radiation-induced $\mathrm{G} 2 / \mathrm{M}$ arrest. Biochim Biophys Acta 1833: 2911-2921, 2013.

19. Miyata H, Doki Y, Yamamoto $\mathrm{H}$, et al: Overexpression of CDC25B overrides radiation-induced G2-M arrest and results in increased apoptosis in esophageal cancer cells. Cancer Res 61: 3188-3193, 2001.

20. Rodemann HP: Molecular radiation biology: perspectives for radiation oncology. Radiother Oncol 92: 293-298, 2009.

21. Huang F, Motlekar NA, Burgwin CM, Napper AD, Diamond SL and Mazin AV: Identification of specific inhibitors of human RAD51 recombinase using high-throughput screening. ACS Chem Biol 6: 628-635, 2011.

22. Budke B, Logan HL, Kalin JH, et al: RI-1: a chemical inhibitor of RAD51 that disrupts homologous recombination in human cells. Nucleic Acids Res 40: 7347-7357, 2012.

23. Eisenberg-Lerner A and Kimchi A: The paradox of autophagy and its implication in cancer etiology and therapy. Apoptosis 14: 376-391, 2009.

24. Tsuchihara K, Fujii S and Esumi H: Autophagy and cancer: dynamism of the metabolism of tumor cells and tissues. Cancer Lett 278: 130-138, 2009.

25. White E: Deconvoluting the context-dependent role for autophagy in cancer. Nat Rev Cancer 12: 401-410, 2012.

26. Yenigun VB, Ozpolat B and Kose GT: Response of CD $44^{+} / \mathrm{CD} 24^{-/ \text {low }}$ breast cancer stem/progenitor cells to tamoxifen- and doxorubicin-induced autophagy. Int J Mol Med 31: 1477-1483, 2013.

27. Tekirdag KA, Korkmaz G, Ozturk DG, Agami R and Gozuacik D: MIR181A regulates starvation- and rapamycin-induced autophagy through targeting of ATG5. Autophagy 9: 374-385, 2013.

28. Zhou Y, Wang HD, Zhu L, et al: Knockdown of Nrf2 enhances autophagy induced by temozolomide in U251 human glioma cell line. Oncol Rep 29: 394-400, 2013.

29. Liang N, Jia L, Liu Y, et al: ATM pathway is essential for ionizing radiation-induced autophagy. Cell Signal 25: 2530-2539, 2013.

30. Chaachouay H, Ohneseit P, Toulany M, Kehlbach R, Multhoff G and Rodemann HP: Autophagy contributes to resistance of tumor cells to ionizing radiation. Radiother Oncol 99: 287-292, 2011.

31. Pawlik TM and Keyomarsi K: Role of cell cycle in mediating sensitivity to radiotherapy. Int J Radiat Oncol Biol Phys 59: 928-942, 2004.

32. Corcelle E, Nebout M, Bekri S, et al: Disruption of autophagy at the maturation step by the carcinogen lindane is associated with the sustained mitogen-activated protein kinase/extracellular signal-regulated kinase activity. Cancer Res 66: 6861-6870, 2006.

33. Takenaka T, Yoshino I, Kouso H, et al: Combined evaluation of Rad51 and ERCC1 expressions for sensitivity to platinum agents in non-small cell lung cancer. Int J Cancer 121: 895-900, 2007.

34. Du LQ, Wang Y, Wang H, Cao J, Liu Q and Fan F-Y: Knockdown of Rad51 expression induces radiation- and chemo-sensitivity in osteosarcoma cells. Med Oncol 28: 1481-1487, 2011.

35. Yamamori T, Meike S, Nagane M, Yasui H and Inanami O: ER stress suppresses DNA double-strand break repair and sensitizes tumor cells to ionizing radiation by stimulating proteasomal degradation of Rad51. FEBS Lett 587: 3348-3353, 2013. 